\title{
Gap and Low-Resistance Junctions between Cells in Culture
}

\author{
Dieter F. Hülser and Anthony Demsey * \\ Max-Planck-Institut für Virusforschung, Abteilung für physikalische Biologie \\ und Elektronenmikroskopie-Labor Tübingen, Germany
}

(Z. Naturforsch. 28 c, 603-606 [1973] ; received July 17, 1973)

\begin{abstract}
Cell cultures, low-resistance junction, gap junction, intercellular communication, freeze fracture
Established lines of both fibroblastoid and epithelioid cells have been investigated by electrophysiological and freeze-fracture methods. Between the ionically coupled (by "low-resistance junc. tions") fibroblastoid cells numerous gap junctions were found. Between the non-coupled epithelioid cells gap junctions could not be detected.
\end{abstract}

\section{Introduction}

In vitro cultured cells are connected by different membrane junctions which may serve as a matrix for the monolayer, but which may also enable intercellular communication. Some of these intercellular information exchanges may be detected by ionic coupling between the cells through so-called "low-resistance junctions". Gilula and coworkers ${ }^{1}$, for instance, have shown that metabolic cooperation between inosinic guanylic pyrophosphorylase(IPP)-positive chinese hamster cells and their IPP-negative sublines is only present in ionically coupled fibroblasts, and only between these coupled cells could gap junctions be found. Such gap junctions have also been described by other authors ${ }^{2-5}$ for different ionically coupled fibroblastoid cell types. Recently evidence has been given ${ }^{6}$ for a correlation between the gross morphology of established cell lines and ionic coupling: Fibroblastoid cells are ionically coupled, epithelioid cells are not. We have, therefore, investigated epithelioid cells as well as fibroblastoid cells of normal and malignant origin in order to test whether epithelioid cells also have gap junctions, or whether the correlation between low-resistance junctions and gap junctions could be confirmed. The freeze-fracture technique was employed preferentially to thin sectioning since it allows the examination of large areas of membrane surface. By this method the coupled fibroblastoid cells (3T3 and BICR/ MIR-K) have been shown to possess gap junctions, whereas, with the same method and with as much as a three times longer investigation period, we could

Requests for reprints should be sent to Dr. D. F. Hülser, Max-Plandk-Institut für Virusforschung, Abteilung Physikalische Biologie, D-7400 Tibingen, Spemannstraße 35. not detect any gap junctions in the non-coupled epithelioid cells (HeLa and RE).

\section{Materials and Methods}

\section{Cell cultures}

The mouse-embryo originated fibroblastoid 3T3 cells were obtained from Prof. W. Schäfer, MPI für Virusforschung, Biologisch-Medizinische Abteilung, Tübingen. BICR/M1R-K cells are also fibroblastoid and were derived from a transplantable tumour of the Marshall rat ${ }^{7}$. The epithelioid HeLa cells, originated from a human cervix carcinoma, were kindly provided by Prof. H. Friedrich-Freksa of this department. The epithelioid RE cells were derived from embryonic rats and have grown for about six years permanently in culture ${ }^{8}$.

Culturing of all the cells was performed at $37^{\circ} \mathrm{C}$ in a modified Eagle-Dulbecco medium ${ }^{8}$ supplemented with $10 \%$ calf serum. At the end of the log phase of growth the cells were passaged by separating them at $37^{\circ} \mathrm{C}$ with $0.25 \%$ trypsin in Ca-Mg-free isotonic phosphate-buffered saline (PBS). After washing in medium, the cells used for the electrophysiological experiments were seeded into plastic petri dishes (Falcon or Greiner, Nürtingen; $50 \mathrm{~mm}$ diameter, $1 \cdot 10^{5}$ cells/dish; $5 \mathrm{ml}$ medium). For freeze fracture experiments the cells were grown in Falcon plastic bottles.

\section{Electrical measurements}

The determination of ionic coupling was performed with three Ling-Gerard glass-microelectrodes filled with $3 \mathrm{M} \mathrm{KCl}+2 \mathrm{mM} \mathrm{K}$-citrate (resistance $30-50 \mathrm{M} \Omega$, tip potential $\leqq 5 \mathrm{mV}$ in the modified

\footnotetext{
* Present Address: New York State Department of Health, Division of Laboratories and Research, Clinical Pathology Laboratories, New Scotland Avenue, Albany, New York 12201.
} 
Eagle-Dulbecco medium). Voltages were measured using Keithley 605 negative capacitance electrometers. Constant current pulses were supplied from a Tektronix generator $(601 / 161 / 162)$ via a photoncoupled isolator ${ }^{9}$. During the measurements the cells were observed with a Standard Zeiss phasecontrast microscope. For details see 1. c. ${ }^{10}$.

\section{Freeze fracture}

Cells in Falcon plastic bottles were grown at $37^{\circ} \mathrm{C}$ until confluent. The medium was then carefully poured off, so as not to disturb the cells lying in the monolayer and the cells were fixed in situ at room temperature in $3 \%$ glutaraldehyde in PBS (pH 7.2). After one hour, the glutaraldehyde was carefully decanted and the cells were washed in situ several times with PBS. Finally glycerol solutions in PBS were added in several changes of increasing concentrations to a final glycerol concentration of $30 \%$. After infiltration overnight at $4{ }^{\circ} \mathrm{C}$, the cells were pelleted, and then freeze fractured. (without etching) and replicated in a Balzers $500 \mathrm{M}$ instrument at a stage temperature of $-100^{\circ} \mathrm{C}^{11}$. The $\mathrm{C} / \mathrm{Pt}$ replicas were cleaned in sulfuric acid and eau de javelle and mounted on Pioloform- and carboncoated grids. Before viewing in a Siemens.Elmiskop IA the grids were arranged and coded by a third person, thus avoiding any bias during the examination for gap junctions. Prints are mounted with the black shadows in a downward direction.

\section{Results and Discussion}

The gross morphology of the investigatted established cell lines was determined after the cells had formed a confluent monolayer. The fibroblastoid 3T3 cells (Fig. 1*) have long filaments which tend to grow over neighbouring cells. It is, therefore, difficult to distinguish whether cells are separated by others or not. 3T3 cells are representative of the ionically coupled cell type. The epithelioid HeLa cells (Fig. 2) seem clearly separated from each other; nevertheless their membranes are close together, as could be seen from ultrathin sections or freeze fracture replicas. These epithelioid: cells are representative of the non-coupled cell type. Since the electrical properties of the four investigated cell types have already been described ${ }^{6,12}$, we show here only two oscilloscope readings which demonstrate the states of coupling and non-coupling respectively. In Fig. 3 an example of ionic coupling

\footnotetext{
- Figs 1-5 see Table on page 604 a.
}

can be seen with fibroblastoid 3T3 cells. A $30 \mathrm{nA}$ current pulse injected with a Ling-Gerard microelectrode into one cell results in a $70 \mathrm{mV}$ hyper. polarization pulse measured by a voltage recording electrode in the same cell. The current can simul. taneously spread into the neighbouring cells and a hyperpolarization pulse of about $40 \mathrm{mV}$ can be measured in one of the adjacent cells, indicating the degree of coupling. The epithelioid HeLa cells, however, are hyperpolarized to the same extent as the 3T3 cells with considerably less current - about $15 \mathrm{nA}$. It is impossible to detect simultaneous hyperpolarization pulses in adjacent cells; they are, therefore, considered non-coupled (Fig. 4).

From both coupled and non-coupled cell types a normal line (3T3 and RE respectively) and a malignant line (BICR/M1R-K and HeLa respectively) have been investigated for gap junctions. This type of junction was first described by Revel and Karnovsky ${ }^{13}$ in thin sections of mouse-heart and liver cells by employing a lanthanum stain. Where the membranes of two adjacent cells form a junction between the cells, periodic gaps in the lanthanum precipitate can be observed. A cross section of two such junctions is schematically shown in Fig. 5 a (regions of blackened diamonds). However, since the yield of observed gap junctions is much higher with freeze fracturing - where large expanses of membrane are commonly found - and since a cross section through such a junction is difficult to find in thin-sectioned cell culture material, most of our ultrastructural investigations were carried out using replicas of freeze fractured material. Having examined more than $\mathbf{5 0}$ grids, many of which were viewed twice, and without knowing the origin of the replicas, we had only one false negative finding: $A$ poor preparation of BICR/MIR-K cells revealed no gap junctions. All other preparations of the ionical. ly coupled 3T3 and BICR/MIR-K cells were positive. Gap junctions could easily be found and in most cases, when one gap junction was seen, others were detected in close proximity. In preparations of non-coupled HeLa and RE cells, gap junctions have not been detected even with a three times longer investigation period as compared with the positive preparations.

Fig. $6^{\text {* }}$ is an example of a freeze-fracture preparation: A large expanse of membrane from 3$]^{3} 3$ cells containing several gap junctions. Through Figs

- Figs 6-10 see Table on page 604 b. 


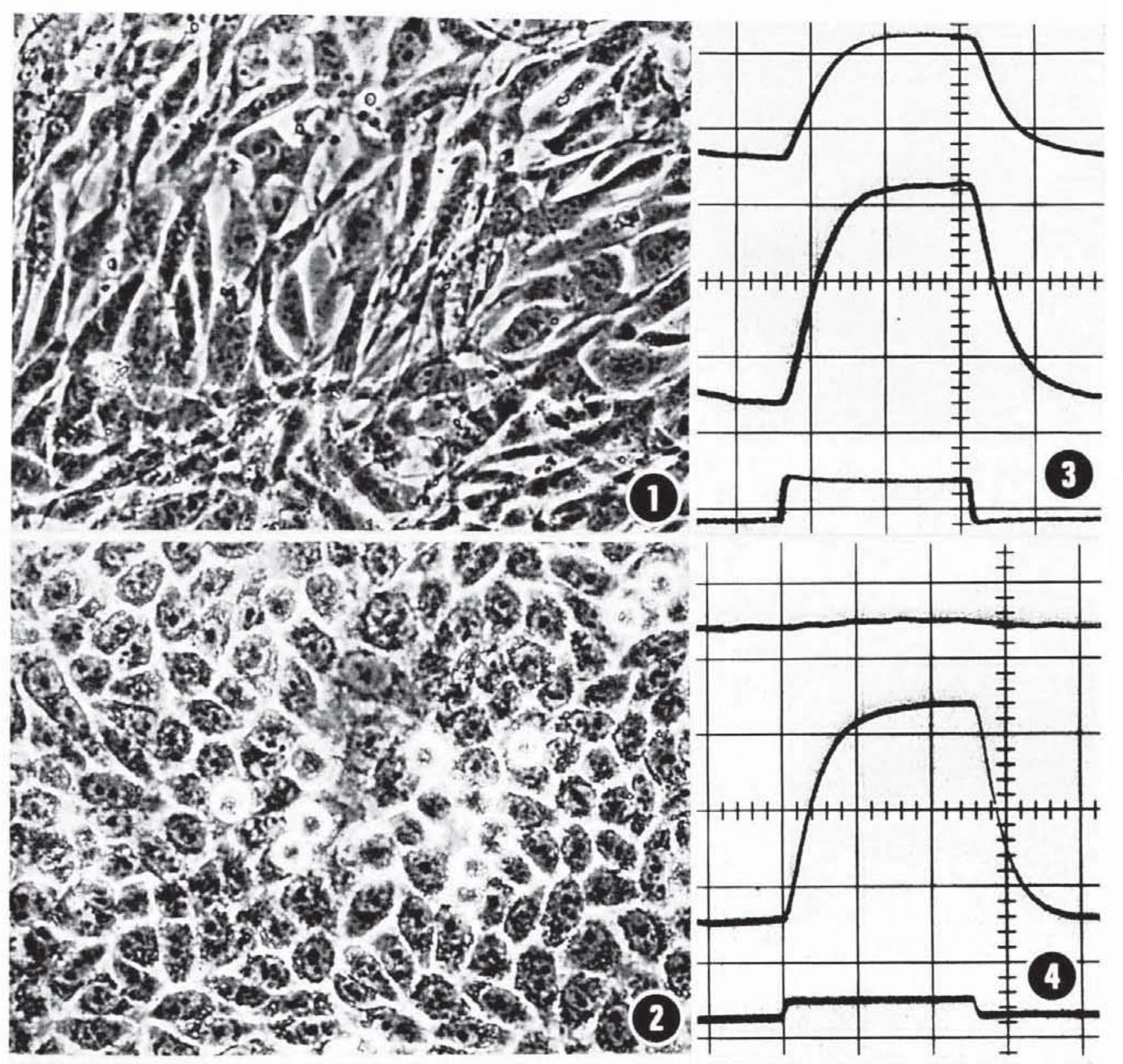

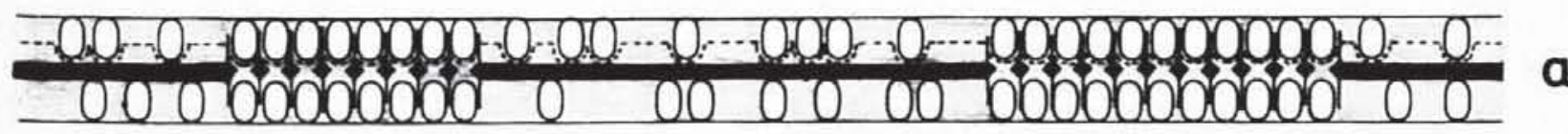
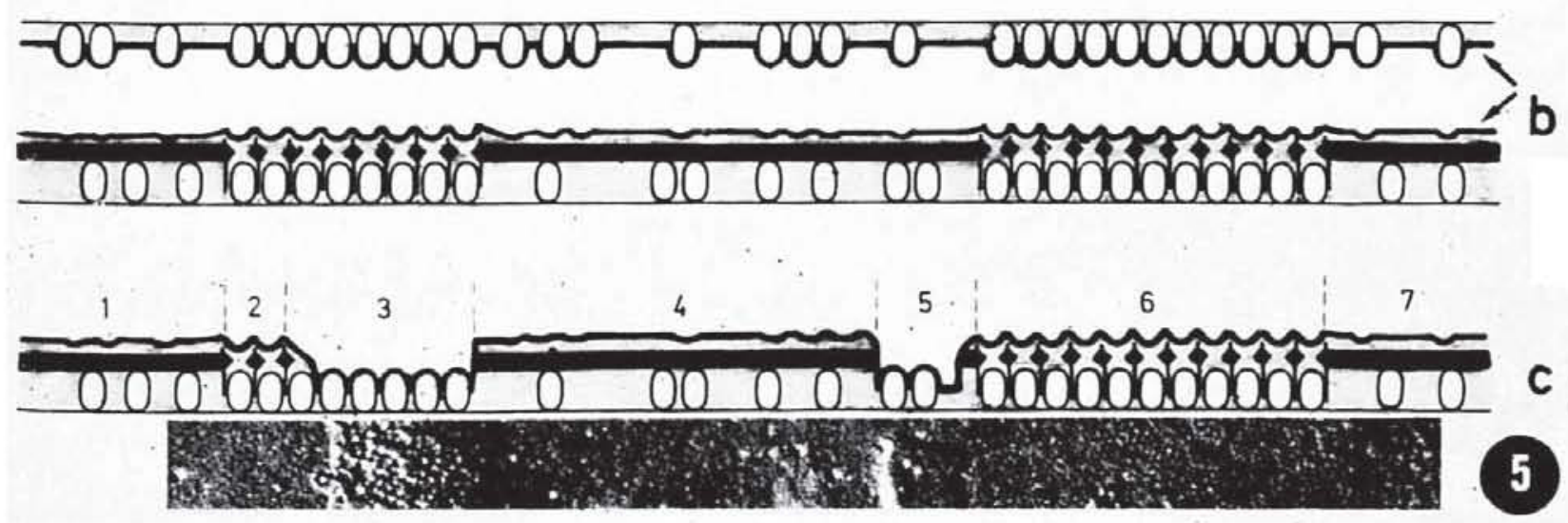

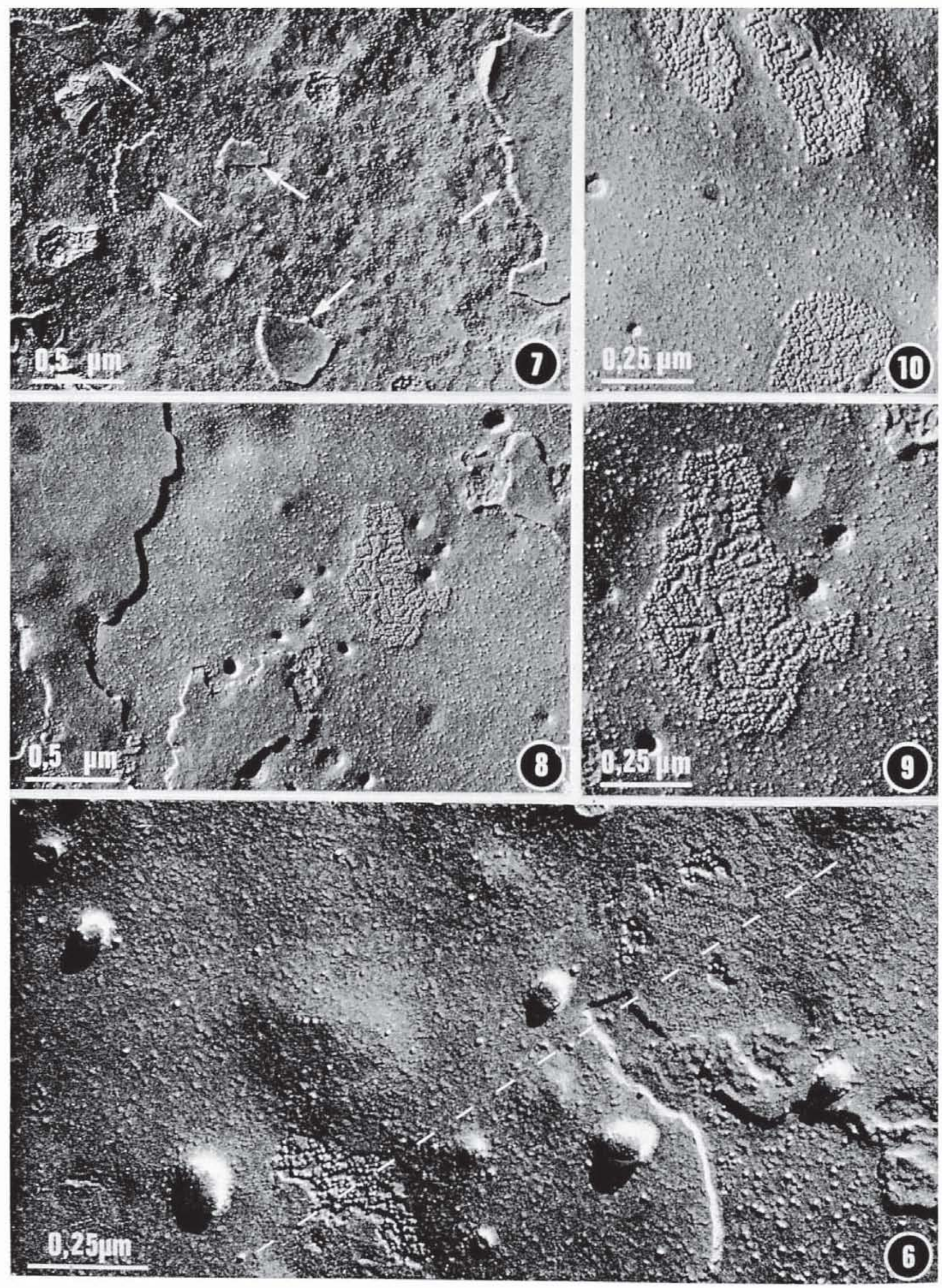
5 and 6 we have attempted to clarify the way in which membranes and particularly gap junctions are visualized using the freeze-fracture technique. A dotted line is drawn in Fig. 6 and a cross section of the double membrane under this line, as it would have appeared before freeze fracturing, is schematically shown in Fig. 5 a. In this drawing, the ovals represent the non-lipid particles within each of the two membranes, the intercellular space is black and in the region of the two junctions the "gap" arrangement (area between diamonds) can be seen as it appears in thin sectioning when lanthanum staining is used. (Although membrane-associated particles are not thought to be entirely uniform ${ }^{14}$, we have drawn them so, in order to simplify the discussion.) In the two junctional regions the membrane particles are seen to be highly concentrated and to fit into a hexagonal "honeycomb" arrangement on the opposite side of the membrane. The "honeycomb" areas of the two apposed membranes lie back-to. back $4,15-18$.

In freeze fracture, membranes tend to break through their middle with most of the membrane particles appearing on one side ${ }^{19-21}$, and in Fig. 5 a the upper membrane shows such an idealized fracture (dotted line). When separated and "replicated" as in freeze fracturing (Fig. $5 \mathrm{~b}$ ), either of the two typical freeze-fracture membrane surfaces can be found: An outer-facing, highly particulated, " $A$ " surface (top arrow); and an inner-facing, sparsely particulated, "B" surface (bottom arrow). The highly concentrated particles and the "honeycomb" would, therefore, be the two characteristic aspects of gap junctions seen in freeze fracture preparations: The concentrated particles being characteristic of that part of the gap junction associated with an "A" surface, and the "honeycomb" being characteristic of that part of the gap junction associated with a "B" surface. The fracture seen in Figs $5 \mathrm{a}$ and $5 \mathrm{~b}$ is of course a highly simplified case. In reality, Fig. 6 shows a fracture which reveals both types of membrane surfaces at one time: The outer-facing (" $A$ ") surface within the membrane of one cell is at the lower right; the remainder is the inner-facing (" $\mathrm{B}$ ") surface within the membrane of the adjacent cell. The two most prominent gap junctions are those indicated by the "honeycomb" in the upper right and the combination of particles and "honeycomb" in the lower left.

The fracture of the membrane in Fig. 6 which lies under the broken line is further schematically seen in Fig. 5 c. An inset of the region in Fig. 6 under discussion is provided at the bottom of Fig. $5 \mathrm{c}$. The apposed (unreplicated) half of the fracture has not been drawn. Starting from the left of Fig. 5 c, which corresponds to the region of the large gap junction in the lower left of Fig. 6, one can trace the fracture as it

1 . begins by revealing the normal " $B$ " surface part of the upper membrane;

2. enters the gap junction (small "honeycomb");

3 . drops down to the area of high particle concentration on the lower membrane " $\mathrm{A}$ " surface be-
Fig. 1. Phase-contrast picture of fibroblastoid 3T3 cells. Magnification $\sim 300 \times$.

Fig. 2. Phase-contrast picture of epithelioid HeLa cells. Magnification $\sim 300 x$.

Fig. 3. Oscillograph tracing of a coupling experiment between 3T3 cells. A $30 \mathrm{nA}$ current pulse of about $20 \mathrm{msec}$ duration (lower trace) results in a $70 \mathrm{mV}$ hyperpolarization in the same cell (middle trace) and in a $40 \mathrm{mV}$ hyperpolarization in one of the adjacent cells (upper trace).

Fig. 4. Oscillograph tracing of the same experiment as shown in Fig. 3, but between HeLa cells. A smaller current pulse (about $15 \mathrm{nA}$ ) results in the same hyperpolarization of about $70 \mathrm{mV}$ and no hyperpolarization can be detected in the adjacent cells. These cells are not ionically coupled.

Fig. 5. a. Schematic representation of two apposed cell mem. branes in cross section which are joined at two points by gap junctions (blackened diamonds). Broken line through upper membrane is an idealized fracture.

b. The two sides of the fracture shown in a. have been pulled apart and "replicated" (arrows) yielding an " $\mathrm{A}$ " surface (upper) and "B" surface (lower). c. Appearance of an actual fracture modelled after a small section of Fig. 6 (compare inset below with broken line on Fig. 6). Further explanation is found in the text.

Fig. 6. Freeze fracture preparation of 3T3 cells showing membranous area with gap junctions on " $A$ " and " $B$ " surfaces. Broken line indicates area used as model for Fig. 5 (compare with inset, Fig. 5 c). $\times 80,000$.

Fig. 7. Freeze fracture preparation of HeLa cells showing membranous area of two closely apposed cells. Arrows indicate parts of one cell's membrane ("B" surface) which lie on

the membrane (" $A$ " surface) of the other cell. $\times 30,000$.

Fig. 8. Freeze fracture preparation of $3 T 3$ cells with a gap junction lying on one of the membrane surface (" $A$ "). $\times 30,000$.

Fig. 9. Higher magnification of gap junction seen in Fig. 8. $\times 60,000$.

Fig. 10. Freeze fracture preparation of BICR/MIR-K cells showing a membrane surface (" $A$ ") with several gap junctions. $\times 60,000$. 
fore rising to the normal " $\mathrm{B}$ " surface of the upper membrane and leaving the gap junction;

4. passes over more of the upper membrane's normal "B" surface;

5. drops down to the "A" surface of the lower membrane and passes over two small particles before rising again to the " $\mathrm{B}$ " surface of the upper membrane;

6. enters a large junctional area corresponding to the "honeycomb" in the upper right of Fig. 6;

7. finally leaves the gap junction and again passes over normal "B" surface membrane.

An example of a freeze-fractured HeLa cell preparation is seen in Fig. 7. Most of the area seen is the "A" surface of one cell. (Notice the large num. ber of scattered membrane particles.) Lying on top of this surface are several patches of the "B" surface (arrows) from a closely apposed cell. (Only a few membrane particles are scattered on this surface.) As is typical of HeLa cells, no gap junctions are seen, although the cells certainly appear to be close enough for gap junction formation. Fig. 8 is an example of 3T3 cell membrane surfaces quite similar in nature to those of the HeLa cells seen in Fig. 7, and printed at the same magnification. The two membrane surface types, " $A$ " and " $B$ ", are evident, but lying on the " $A$ " surface is the large cluster of particles characteristic of gap junctions. A higher magnification of this gap junction is seen in Fig. 9. Such junctional areas were also easily found in BICR/M1R-K cells. An example is shown in Fig. 10. It is interesting to note that the concentration of the usual random membrane particles

1 N. B. Gilula, O. R. Reeves, and A. Steinbach, Nature [London] 235, 262 [1972].

2 R. G. Johnson, J. Cell Biol. 55, 126 a [1972].

3 R. G. Johnson and J. D. Sheridan, Science [Washington] 174, 717 [1971].

4. Pinto da Silva and N. B. Gilula, Exp. Cell Res. 71, 393 [1972].

5 J. P. Revel, A. G. Yee, and A. J. Hudspeth, Proc. nat. Acad. Sci. USA 68, 2924 [1971].

B D. F. Hülser and D. J.Webb, Exp. Cell Res. 80, 210 [1973].

7 M. F. Rajewsky and A. Grüneisen, Eur. J. Immunol. 2, 445 [1972].

8 W. Frank, H.-J. Ristow, and S. Schwalb, Exp. Cell Res. 70, 390 [1972].

I. Baird, Med. Biol. Engng. 5, 295 [1967].

10 D. F. Hülser, Methods in Cell Physiology 8 [1973], in press.

11 H. Moor, Philos. Trans. Roy. Soc. Lond., Ser. B 261, 12] [1971]. lying on the " $A$ " surface of split membranes varies greatly with the different cell lines. Not only the HeLa cells (Fig. 7) but also the RE cells generally display a uniformly high concentration of these particles. The 3T3 cells (Figs 8 and 9), on the other hand, have a relatively sparse concentration of particles over the non-junctional regions and the BICR/M1R-K cells (Fig. 10) have an even sparser concentration. One plausible explanation for this, which is consistent with the fluid mosaic model of the cell membrane ${ }^{22}$, is that particles migrate to the sites of gap junction formation, depleting the concentration of particles on the non-junctional membrane surfaces. Such a difference between fibroblastoid and epithelioid cells of established cultures would also coincide with their observed differences in permeability due to varying bicarbonate concentrations ${ }^{12}$ and with their differences in sensitivity to ouabain ${ }^{23}$.

With the demonstration of gap junctions in fibroblastoid cell lines we have confirmed and expanded upon the findings of other authors ${ }^{1-5}$ that gap junctions can be found in cells which have lowresistance junctions. Furthermore we have presented examples of non-coupled epithelioid cells which have no gap junctions, thus strengthening the idea of an identity between low-resistance junctions and gap junctions.

We wish to thank Miss I. Klein, Mrs. R. MacWil. liams, Mrs. U. Michel, Miss R. Szabo, Mrs. I. Thiering, Mr. G. Berger, and Mr. D. Webb for their excellent technical assistance. This investigation was supported by the Deutsche Forschungsgemeinschaft.

12 D. F. Hülser, Pflügers Arch. Eur. J. Physiol. 325, 174 [1971].

13 J. P. Revel and M. J. Karnovsky, J. Cell Biol. 33, C7 [1967].

14 H. W. Meyer and H. Winkelmann, Protoplasma 75, 255 [1972].

15 J. P. Chalcroft and S. Bullivant, J. Cell Biol. 47, 49 [1970].

16 N. S. McNutt and R. S. Weinstein, J. Cell Biol. 47, 666 [1970].

17 R. L. Steere and J. R. Sommer, J. de Microsc. 15, 205 [1972].

18 E. L. Benedetti, I. Dunia, and A. Diawara, Eur. J. Cancer 9, 263 [1973].

19 D. Branton, Proc. nat. Acad. Sci. USA 55, 1048 [1966].

20 D. Branton, Annu. Rev. Plant Physiol. 20, 209 [1969].

21 H. W. Meyer and H. Winkelmann, Protoplasma 68, 253 [1969].

22 S. J. Singer and G. L. Nicolson, Science [Washington] 175,720 [1972]

2s D. F. Hülser and D. J. Webb, unpublished results. 